\begin{tabular}{|r|l|}
\hline \multicolumn{2}{|c|}{ Statistica Sinica Preprint No: SS-2020-0352 } \\
\hline Title & $\begin{array}{l}\text { Scaled Partial Envelope Model in Multivariate Linear } \\
\text { Regression }\end{array}$ \\
\hline Manuscript ID & SS-2020-0352 \\
\hline URL & http://www.stat.sinica.edu.tw/statistica/ \\
\hline DOI & $10.5705 /$ ss.202020.0352 \\
\hline Complete List of Authors & $\begin{array}{l}\text { Jing Zhang, } \\
\text { Zhensheng Huang and } \\
\end{array}$ \\
\hline Lixing Zhu \\
\hline Corresponding Author & Zhensheng Huang \\
\hline E-mail & stahzs@126.com \\
\hline Notice: Accepted version subject to English editing. \\
\hline
\end{tabular}


Statistica Sinica

\title{
Scaled Partial Envelope Model in Multivariate Linear Regression
}

\author{
Jing Zhang ${ }^{a, b}$, Zhensheng Huang ${ }^{a}$ and Lixing $\mathrm{Zhu}^{c, d}$ \\ ${ }^{a}$ School of Science, Nanjing University of Science and Technology \\ ${ }^{b}$ School of Finance, Chuzhou University \\ ${ }^{c}$ Center for Statistics and Data Science, Beijing Normal University \\ ${ }^{d}$ Department of Mathematics, Hong Kong Baptist University
}

Abstract:

Inference which is based on the partial envelope model is variational or nonequivariant under rescaling of the responses, and it is inclined to restrict utilization to responses that are measured in the identical or analogous units. The efficiency acquisitions promised by partial envelopes frequently cannot be accomplished when the responses are measured in diverse scales. Based on the research results of Cook and Su (2013), we extend the partial envelope model to the scaled partial envelope model which can overcome the disadvantage above and enlarge the scope of partial envelopes. It maintains the potential of the partial envelope model to add up efficiency and is invariable to scale changes. Further, we demonstrate the maximum likelihood estimators and their properties. At last, simulation studies and real data example demonstrate that the advantage 
of scaled partial envelope estimators, with the comparison to the standard model estimators, the partial envelope estimators and the scaled envelope estimators.

Key words and phrases: Dimension reduction; Grassmannian; Scaled envelope model; Partial envelope model; Scale invariance.

\section{Introduction}

The standard multivariate linear regression model which has $p \times 1$ nonstochastic predictor $X$ and $r \times 1$ stochastic response $Y$ can be represented as

$$
Y=\alpha+\beta X+\varepsilon
$$

where $\alpha \in \mathbb{R}^{r}$ is the unknown intercept, $\beta \in \mathbb{R}^{r \times p}$ is the unknown coefficient matrix, the error vector $\varepsilon$ has mean zero and covariance matrix $\Sigma>0$, and it is independent of $X$. The data involve $n$ independent values $Y_{i}$ of $Y$ which are observed at corresponding values $X_{i}$ of $X(i=1, \ldots, n)$. In general, we assume that the predictor is centered in the sample. The model is a cornerstone of multivariate statistics, where studying the interrelation between $X$ and $Y$ through the regression coefficient matrix $\beta \in \mathbb{R}^{r \times p}$ is the focus of this paper. So our interest lies in the estimation of $\beta$.

Cook et al. (2010) introduced response envelopes, and employed a subspace to envelop the material information and eliminate the immateri- 
al variation. This course can result in massive efficiency gains when the immaterial variation is larger than the material variation. Based on the development of Cook et al. (2010), several papers have extended the idea of enveloping to more general settings, and have proposed new models to accomplish greater efficiency gains (Su and Cook (2011, 2012, 2013), Cook et al. (2013), Cook and Zhang (2015, 2016, 2018), Khare et al. (2017), Li and Zhang (2017), Zhang and Li (2017), Ding and Cook (2018), Pan et al. (2019), Zhu and Su (2020)).

The partial envelope model introduced by $\mathrm{Su}$ and Cook (2011) results in a parsimonious method for multivariate linear regression when some of the predictors are of special interest. In contrast to the standard model, it has the potential to accomplish extensive efficiency gains in the estimation of the coefficients for the chosen predictors. The partial envelope model is a variation on the envelope model proposed by Cook et al. (2010), but it pays close attention to part of the predictors. It has looser restrictions and can further enhance efficiency. The envelope estimator decreases to the standard estimator when $r \leq p$ and $\beta$ has rank $r$, there is no probability for efficiency acquisitions in this background. Nevertheless, partial envelope removes this restriction, offering gains even when $r \leq p$.

The scaled envelope model proposed by Cook and Su (2013) is scale- 
unchanging and can accomplish efficiency gains except those probability from the initial envelope model. This is achieved by incorporating a scaling matrix into the model, so scale transformations are taken into account during estimation. It maintains the potential of the original envelope methods to add up efficiency and is invariant to scale variations. Cook and $\mathrm{Su}$ (2016) employed the relationship between partial least squares and envelopes to exploit a new method-scaled predictor envelopes that involve predictor scaling into partial least squares type applications. By means of estimating the suitable scales, the scaled predictor envelope model estimators can provide efficiency gains and surpass those offered by partial least squares, and further decrease prediction errors.

The scaled envelope model pays close attention to all predictors, but we can focus on part of the predictors, looser restrictions and further improve efficiency. Our research is motivated by the study of Cook and Su (2013). In order to further improve the efficiency of parameter estimation and keep the scale invariant, we firstly absorb the dimension reduction ideas of partial envelope model to center on some predictors of special interest. Further, we combine the partial envelopes with the scaled envelopes to form the scaled partial envelope model which can increase efficiency availably. The scaled partial envelope model estimators have shown promising performance in 
the simulation studies and real data analysis. From the simulation results, when the envelope subspace is equal to the full space, we can find that the standard model and the scaled envelope model have the same standard deviations, and see that the scaled partial envelope estimators have a remarkable efficiency gain over the partial envelope estimators, and the partial envelope estimators have an obvious efficiency gain over the ordinary least squares estimators and the scaled envelope estimators whether it is under normal error or non-normal error. In the real data analysis, we also see the advantages of the scaled partial envelope model. When the part of response variable $Y$ that is material to all predictors is no less variable than the immaterial part, but the part of response variable $Y$ that is material to part of predictors is much less variable than the immaterial part, the scaled partial envelope estimators have significant advantage over the standard model estimators, the partial envelope estimators and the scaled envelope estimators.

The rest of the paper is organized as follows. Section 2 reviews the envelope model, partial envelope model and scaled envelope model. Further, the scaled partial envelope model is proposed. Section 3 demonstrates maximum likelihood estimators and identifiability for scaled partial envelope model parameters. Section 4 exhibits theoretical properties of the scaled 
partial envelope estimator. Section 5 shows selection of partial envelope dimension $u_{1}$. Simulation studies are carried out to compare our proposed scaled partial envelope model with the standard model, the partial envelope model and the scaled envelope model in Section 6. Real data example is given in Section 7. Some remarks are displayed in Section 8. The proofs of the propositions are provided in the part of Supplementary Materials.

\section{Scaled partial envelope model}

\subsection{Review of envelope model and partial envelope model}

The envelope model (Cook et al., 2010) aims to find a smallest subspace $\mathcal{E} \subseteq \mathbb{R}^{r}$ that satisfies the following two conditions

$$
(a) Q_{\mathcal{S}} Y\left|X \sim Q_{\mathcal{S}} Y,(b) Q_{\mathcal{S}} Y \amalg P_{\mathcal{S}} Y\right| X .
$$

The sign ' $\sim$ ' means identically distributed, and the sign ' $\amalg$ ' means statistically independent. The symbol $P_{(\cdot)}$ projects onto the subspace and $Q=I_{r}-P$. The Property (a) implies that the distribution of $Q_{\mathcal{S}} Y$ does not rely on $X$, so $Q_{\mathcal{S}} Y$ has no information about $\beta$. The Property (b) implies that $Q_{\mathcal{S}} Y$ is conditionally independent of $P_{\mathcal{S}} Y$ given $X$ and thus $Q_{\mathcal{S}} Y$ cannot transmit information about $\beta$ via a link with $P_{\mathcal{S}} Y$. The properties provided by $(2.1)$ equal to the condition $Q_{\mathcal{S}} Y \mid\left(P_{\mathcal{S}}, X\right) \sim Q_{\mathcal{S}} Y$. This ex- 
2.1 Review of envelope model and partial envelope model

pression signifies that the projection $Q_{\mathcal{S}} Y$ is not material to the estimation of $\beta$. All of the immaterial information in $Y$ can be gained by detecting the smallest subspace $\mathcal{S}$ that meets the requirements in (2.1).

Let $\mathcal{B}=\operatorname{span}(\beta)$. Cook et al. (2010) displayed that the pair of conditions (2.1) is equal to the following two conditions

$$
(2 a) \mathcal{B} \subseteq \mathcal{S},(2 b) \Sigma=P_{\mathcal{S}} \Sigma P_{\mathcal{S}}+Q_{\mathcal{S}} \Sigma Q_{\mathcal{S}}
$$

Condition (2b) holds if and only if $P_{\mathcal{S}} Y$ and $Q_{\mathcal{S}} Y$ are uncorrelated given $X$, and it is equivalent to claiming that $\mathcal{S}$ is a reducing subspace of $\Sigma$. These conditions signify that all of the immaterial information can be obtained by choosing $\mathcal{S}$ to be the intersection of all reducing subspaces of $\Sigma$ that include $\mathcal{B}$, which is called the $\Sigma$-envelope of $\mathcal{B}$ and denoted by $\mathcal{E}_{\Sigma}(\mathcal{B})$ or shortened to $\mathcal{E}$.

Let $u=\operatorname{dim}\left\{\mathcal{E}_{\Sigma}(\mathcal{B})\right\}$, and let $\Gamma \in \mathbb{R}^{r \times u}$ and $\Gamma_{0} \in \mathbb{R}^{r \times(r-u)}$ denote semiorthogonal basis matrices for $\mathcal{E}_{\Sigma}(\mathcal{B})$ and its orthogonal complement $\mathcal{E}_{\Sigma}^{\perp}(\mathcal{B})$ respectively. By means of forcing conditions (2.2) on the standard model (1.1), the coordinate form of the envelope model can be acquired as follows:

$$
Y=\alpha+\Gamma \eta X+\varepsilon, \Sigma=\Gamma \Omega \Gamma^{T}+\Gamma_{0} \Omega_{0} \Gamma_{0}^{T}
$$

where $\eta \in \mathbb{R}^{u \times p}$ is the coordinates of $\beta$ which is relative to $\Gamma$, and $\Omega \in \mathbb{R}^{u \times u}$ and $\Omega_{0} \in \mathbb{R}^{(r-u) \times(r-u)}$ are both positive definite matrices. In the model 
2.1 Review of envelope model and partial envelope model

$(2.3), \mathcal{E}_{\Sigma}(\mathcal{B})$ contacts the mean and covariance matrices and this connection offers the efficiency gains. It can be excellent harvest when variation of the immaterial part $\Gamma_{0}^{T} Y$ is mainly larger than that of the material part $\Gamma^{T} Y$. The parameters in (2.3) are obtained by maximizing a normal likelihood function. Let $\widetilde{\Sigma}_{Y}, \widetilde{\beta}$ and $\widetilde{\Sigma}_{\text {res }}$ denote the sample covariance matrix of $Y$, the least squares estimator of $\beta$, and the sample covariance matrix of the residuals from the least squares regression of $Y$ on $X$ separately. The estimator of the envelope subspace is the span of $\arg \min \left\{\log \left|\Gamma^{T} \widetilde{\Sigma}_{\mathrm{res}} \Gamma\right|+\log \left|\Gamma^{T} \widetilde{\Sigma}_{Y}^{-1} \Gamma\right|\right\}$, and its minimization is the $r \times u$ Grassmannian (Edelman et al., 1998; Adragni et al., 2012).

On the basis of the envelope model, Su and Cook (2011) extended the envelope model to the partial envelope model. The partial envelopes center on the coefficients corresponding to the predictors of interest. Partition $X$ into two sets of predictors $X_{1} \in \mathbb{R}^{p_{1}}$ and $X_{2} \in \mathbb{R}^{p_{2}}, p_{1}+p_{2}=p, p_{1}<r$, and partition accordingly the columns of $\beta$ into $\beta_{1}$ and $\beta_{2}$. Then the model (1.1) can be rewritten as $Y=\alpha+\beta_{1} X_{1}+\beta_{2} X_{2}+\varepsilon$, where $\beta_{1}$ corresponds to the coefficients of interest. The $\Sigma$-envelope for $\mathcal{B}_{1}=\operatorname{span}\left(\beta_{1}\right)$ is mainly taken into account, leaving $\beta_{2}$ as an unconstrained parameter. This results in the parametric structure $\mathcal{B}_{1} \subseteq \mathcal{E}_{\Sigma}\left(\mathcal{B}_{1}\right)$ and $\Sigma=P_{\mathcal{E}, 1} \Sigma P_{\mathcal{E}, 1}+Q_{\mathcal{E}, 1} \Sigma Q_{\mathcal{E}, 1}$, where $P_{\mathcal{E}, 1}$ denotes the projection onto $\mathcal{E}_{\Sigma}\left(\mathcal{B}_{1}\right)$ called the partial envelope 
2.1 Review of envelope model and partial envelope model

for $\mathcal{B}_{1}$. This is identical to the envelope structure, except the partial envelop is correlated with $\mathcal{B}_{1}$ instead of the larger space $\mathcal{B}$. In order to stress on the partial envelope, $\mathcal{E}_{\Sigma}(\mathcal{B})$ is considered as the full envelope. Because of $\mathcal{B}_{1} \subseteq$ $\mathcal{B}$, the partial envelope is included in the full envelope, $\mathcal{E}_{\Sigma}\left(\mathcal{B}_{1}\right) \subseteq \mathcal{E}_{\Sigma}(\mathcal{B})$ which permits the partial envelope to provide gains that may be impossible with the full envelope.

Let $\Gamma \in \mathbb{R}^{r \times u_{1}}$ be a semi-orthogonal matrix with $\Gamma^{T} \Gamma=I_{u_{1}}$, whose columns form a basis for $\mathcal{E}_{\Sigma}\left(\mathcal{B}_{1}\right)$. Let $\left(\Gamma, \Gamma_{0}\right) \in \mathbb{R}^{r \times r}$ be an orthogonal matrix and $\eta \in \mathbb{R}^{u_{1} \times p_{1}}$ be the coordinates of $\beta_{1}$ which is related to the basis matrix $\Gamma$. Then, the coordinate version of the partial envelope model can be expressed as follows

$$
Y=\alpha+\Gamma \eta X_{1}+\beta_{2} X_{2}+\varepsilon, \Sigma=\Gamma \Omega \Gamma^{T}+\Gamma_{0} \Omega_{0} \Gamma_{0}^{T},
$$

where $\Omega \in \mathbb{R}^{u_{1} \times u_{1}}$ and $\Omega_{0} \in \mathbb{R}^{\left(r-u_{1}\right) \times\left(r-u_{1}\right)}$ are both positive definite matrices, and they serve as coordinates of $\Sigma_{\mathcal{E}_{1}}$ and $\Sigma_{\mathcal{E}_{1}^{\perp}}$ separately which are related to the basis matrices $\Gamma$ for $\mathcal{E}_{\Sigma}\left(\mathcal{B}_{1}\right)$ and its orthogonal complement.

To explain it better, let $R_{1 \mid 2}$ denote the population residuals from the multivariate linear regression of $X_{1}$ on $X_{2}$. The predictor $X$ is required to be centered. Then, the linear model can be re-parameterized as $Y=$ $\alpha+\beta_{1} R_{1 \mid 2}+\beta_{2}^{*} X_{2}+\varepsilon$, where $\beta_{2}^{*}$ is a linear combination of $\beta_{1}$ and $\beta_{2}$. Next, let $R_{Y \mid 2}=Y-\alpha-\beta_{2}^{*} X_{2}$, the population residuals from the regression 
2.2 Review of scaled envelope model

of $Y$ on $X_{2}$ alone. A linear model which contains $\beta_{1}$ alone is written as: $R_{Y \mid 2}=\beta_{1} R_{1 \mid 2}+\varepsilon$. The partial envelope $\mathcal{E}_{\Sigma}\left(\mathcal{B}_{1}\right)$ is identical to the full envelope for $\mathcal{B}_{1}$ in the regression of $R_{Y \mid 2}$ on $R_{1 \mid 2}$. That is to say, the partial envelope can be interpreted in the light of $Q_{\mathcal{S}} Y\left|X \sim Q_{\mathcal{S}} Y, Q_{\mathcal{S}} Y \amalg P_{\mathcal{S}} Y\right| X$ which is applied to the regression of $R_{Y \mid 2}$ on $R_{1 \mid 2}$. The predictors have been centered, so the maximum likelihood estimator of $\alpha$ is simply $\widehat{\alpha}=\bar{Y}$. The estimators of the remaining parameters require the estimator of $\mathcal{E}_{\Sigma}\left(\mathcal{B}_{1}\right)$.

\subsection{Review of scaled envelope model}

The envelope model (2.3) is not invariant or equivariant under linear transformations of the response. Assume that $Y$ is rescaled via multiplication by a nonsingular diagonal matrix $A$. Let $Y_{N}=A Y$ denote the new response, let $\widehat{\beta}$ and $\widehat{\Sigma}$ denote the estimators of $\beta$ and $\Sigma$ based on the envelope model for $Y$ on $X$ separately, and let $\widehat{\beta}_{N}$ and $\widehat{\Sigma}_{N}$ denote the estimators of $\beta$ and $\Sigma$ based on the envelope model for $Y_{N}$ on $X$ respectively. Accordingly, there is usually no invariance, for example, $\widehat{\beta}_{N}=\widehat{\beta}, \widehat{\Sigma}_{N}=\widehat{\Sigma}$. Or equivalently, for instance, $\widehat{\beta}_{N}=A \widehat{\beta}, \widehat{\Sigma}_{N}=A \widehat{\Sigma} A$. As a matter of fact, the dimension of the envelope subspace may alter on account of the transformation. Based on the above motivation and thinking, Cook and Su (2013) extended the envelope model to the scaled envelope model. 
2.3 Scaled partial envelope model

For the sake of exhibiting a rescaling, a diagonal matrix $\Lambda=\operatorname{diag}\left\{1, \lambda_{2}\right.$, $\left.\ldots, \lambda_{r}\right\} \in \mathbb{R}^{r \times r}$ is introduced with $\lambda_{i}>0$ for $i=2, \ldots, r$, such that $Y_{N}=$ $\Lambda^{-1} Y$ follows an envelope model where the dimension of the envelope subspace $\mathcal{E}_{\Lambda^{-1} \Sigma \Lambda^{-1}}\left(\Lambda^{-1} \mathcal{B}\right)$ is equivalent to $u$. As a result, $\Lambda^{-1} \mathcal{B} \subseteq \operatorname{span}(\Gamma)$, and $\Lambda^{-1} \Sigma \Lambda^{-1}=P_{\Gamma} \Lambda^{-1} \Sigma \Lambda^{-1} P_{\Gamma}+Q_{\Gamma} \Lambda^{-1} \Sigma \Lambda^{-1} Q_{\Gamma}$, where $\Gamma \in \mathbb{R}^{r \times u}$ is an orthogonal basis of $\mathcal{E}_{\Lambda^{-1 \Sigma} \Lambda^{-1}}\left(\Lambda^{-1} \mathcal{B}\right)$ and $\Gamma_{0} \in \mathbb{R}^{r \times(r-u)}$ is a completion of $\Gamma$.

The coordinate form of the scaled envelope model is as follows

$$
Y=\alpha+\Lambda \Gamma \eta X+\varepsilon, \Sigma=\Sigma_{\mathcal{E}}+\Sigma_{\mathcal{E}^{\perp}}=\Lambda \Gamma \Omega \Gamma^{T} \Lambda+\Lambda \Gamma_{0} \Omega_{0} \Gamma_{0}^{T} \Lambda
$$

Here $\beta=\Lambda \Gamma \eta$ with $\eta=\Gamma^{T} \Lambda^{-1} \beta \in \mathbb{R}^{u \times p}$ is the coefficient matrix, and $\Omega=\operatorname{var}\left(\Gamma^{T} \Lambda^{-1} Y\right)=\Gamma^{T} \Lambda^{-1} \Sigma \Lambda^{-1} \Gamma \in \mathbb{R}^{u \times u}$ and $\Omega_{0}=\operatorname{var}\left(\Gamma_{0}^{T} \Lambda^{-1} Y\right)=$ $\Gamma_{0}^{T} \Lambda^{-1} \Sigma \Lambda^{-1} \Gamma_{0} \in \mathbb{R}^{(r-u) \times(r-u)}$ are both positive definite matrices.

\subsection{Scaled partial envelope model}

Now we discuss the case $n>\max (r, p)$. A diagonal matrix $\Lambda=$ $\operatorname{diag}\left\{1, \lambda_{2}, \ldots, \lambda_{r}\right\} \in \mathbb{R}^{r \times r}$ with $\lambda_{i}>0(i=2, \ldots, r)$ is introduced, so that $(Y)_{N}=\Lambda^{-1} Y$ follows a partial envelope model where the dimension of the partial envelope subspace $\mathcal{E}_{\Lambda^{-1} \Sigma \Lambda^{-1}}\left(\Lambda^{-1} \mathcal{B}_{1}\right)$ is equivalent to $u_{1}$. Hence, $\Lambda^{-1} \mathcal{B}_{1} \subseteq \operatorname{span}(\Gamma)$ and $\Lambda^{-1} \Sigma \Lambda^{-1}=P_{\Gamma} \Lambda^{-1} \Sigma \Lambda^{-1} P_{\Gamma}+Q_{\Gamma} \Lambda^{-1} \Sigma \Lambda^{-1} Q_{\Gamma}$, where $\Gamma \in \mathbb{R}^{r \times u_{1}}$ is an orthogonal basis of $\mathcal{E}_{\Lambda^{-1} \Sigma \Lambda^{-1}}\left(\Lambda^{-1} \mathcal{B}_{1}\right)$ and $\Gamma_{0} \in \mathbb{R}^{r \times\left(r-u_{1}\right)}$ is a completion of $\Gamma$. 
2.3 Scaled partial envelope model

The coordinate form of the scaled partial envelope model is as follows

$$
Y=\alpha+\Lambda \Gamma \eta X_{1}+\beta_{2} X_{2}+\varepsilon, \Sigma=\Sigma_{\mathcal{E}_{1}}+\Sigma_{\mathcal{E}_{1}^{\perp}}=\Lambda \Gamma \Omega \Gamma^{T} \Lambda+\Lambda \Gamma_{0} \Omega_{0} \Gamma_{0}^{T} \Lambda
$$

Here $\beta_{1}=\Lambda \Gamma \eta$ with $\eta=\Gamma^{T} \Lambda^{-1} \beta_{1} \in \mathbb{R}^{u_{1} \times p_{1}}$ is the coefficient matrix, and $\Omega=\operatorname{var}\left(\Gamma^{T} \Lambda^{-1} Y\right)=\Gamma^{T} \Lambda^{-1} \Sigma \Lambda^{-1} \Gamma \in \mathbb{R}^{u_{1} \times u_{1}}$ and $\Omega_{0}=\operatorname{var}\left(\Gamma_{0}^{T} \Lambda^{-1} Y\right)=$ $\Gamma_{0}^{T} \Lambda^{-1} \Sigma \Lambda^{-1} \Gamma_{0} \in \mathbb{R}^{\left(r-u_{1}\right) \times\left(r-u_{1}\right)}$ are both positive definite matrices. A linear model which involves $\beta_{1}$ alone is written as: $R_{Y \mid 2}=\Lambda \Gamma \eta R_{1 \mid 2}+\varepsilon$. In this model, $R_{1 \mid 2}$ denote the population residuals from the multivariate linear regression of $X_{1}$ on $X_{2}$, and $R_{Y \mid 2}=Y-\alpha-\beta_{2}^{*} X_{2}$ denote the population residuals from the regression of $Y$ on $X_{2}$ alone where $\beta_{2}^{*}$ is a linear combination of $\beta_{1}$ and $\beta_{2}$. In order to identify better and compute easily, we set the first element of $\Lambda$ to 1 for the scaling parameters. Otherwise, we can multiply $\Lambda$ by an arbitrary constant $c$ and multiply $\eta$ by its reciprocal $1 / c$.

For a fixed dimension $u_{1}$, the number of parameters in the scaled partial envelope model $(2.6)$ is $\mathcal{N}_{\mathrm{SPE}}=2 r-1+p_{1} u_{1}+p_{2} r+r(r+1) / 2$. This is because, we need $r$ parameters for $\alpha, r-1$ parameters for $\Lambda, p_{1} u_{1}$ parameters for $\eta, p_{2} r$ parameters for $\beta_{2}, u_{1}\left(u_{1}+1\right) / 2$ parameters for $\Omega$, and $\left(r-u_{1}\right)(r-$ $\left.u_{1}+1\right) / 2$ parameters for $\Omega_{0}$. The envelope subspace $\mathcal{E}_{\Lambda^{-1} \Sigma \Lambda^{-1}}\left(\Lambda^{-1} \mathcal{B}_{1}\right)$ is on an $r \times u_{1}$ Grassmann manifold, which is the set of all $u_{1}$ dimensional subspaces in an $r$ dimensional space, so it has $u_{1}\left(r-u_{1}\right)$ free parameters. 
Then we have the following summary for each model:

(i) standard linear model, $\mathcal{N}_{\mathrm{OLS}}=r+p r+r(r+1) / 2($ Cook et al. $(2010))$;

(ii) envelope model, $\mathcal{N}_{\mathrm{E}}=r+p u+r(r+1) / 2($ Cook et al. (2010));

(iii) partial envelope model, $\mathcal{N}_{\mathrm{PE}}=r+p_{1} u_{1}+p_{2} r+r(r+1) / 2(\mathrm{Su}$ and Cook (2011));

(iv) scaled envelope model, $\mathcal{N}_{\mathrm{SE}}=2 r-1+p u+r(r+1) / 2(\mathrm{Cook}$ and $\mathrm{Su}$ $(2013))$

(v) scaled partial envelope model, $\mathcal{N}_{\mathrm{SPE}}=2 r-1+p_{1} u_{1}+p_{2} r+r(r+1) / 2$.

3. Maximum likelihood estimators and identifiability for scaled partial envelope model parameters

\subsection{Maximum likelihood estimators when $\Lambda$ is known}

Here, we discuss maximum likelihood estimation when $\Lambda$ is known.

When $\Lambda=I_{r}$, the subscript ' $O$ ' is employed to indicate quantities in this model to differentiate it from the ordinary partial envelope model (2.4). Under the circumstances, the response $Y$ in (2.6) is converted to $\Lambda^{-1} Y$, 
3.2 Maximum likelihood estimators when $\Lambda$ is unknown14

and then we express the generating partial envelope model as

$$
\left\{\begin{array}{l}
\Lambda^{-1} Y=\alpha_{o}+\Gamma \eta X_{1}+\beta_{2} X_{2}+\varepsilon_{o}, \\
\operatorname{var}\left(\varepsilon_{o}\right)=\Sigma_{o}=\Sigma_{\mathcal{E}_{1}}+\Sigma_{\mathcal{E}_{1}^{\perp}}=\Gamma \Omega \Gamma^{T}+\Gamma_{0} \Omega_{0} \Gamma_{0}^{T} .
\end{array}\right.
$$

Through the above equations, we can obtain the scaled partial envelope

estimators $\widehat{\beta}_{1, \Lambda}$ of $\beta_{1}$ and $\widehat{\Sigma}_{\Lambda}$ of $\Sigma$. When $\Lambda$ is known, we convert $Y$ to $\Lambda^{-1} Y$, and then estimate $\beta_{1, o}=\Gamma \eta$ and $\Sigma_{o}$ in the model (3.1) which follows Su and Cook (2011). Then $\widehat{\beta}_{1, \Lambda}=\Lambda \widehat{\beta}_{1, o}$ and $\widehat{\Sigma_{\Lambda}}=\Lambda \widehat{\Sigma}_{o} \Lambda$. Model (3.1) which has response $\Lambda^{-1} Y$, is simply an ordinary partial envelope model.

\subsection{Maximum likelihood estimators when $\Lambda$ is unknown}

Supposing that the errors $\varepsilon$ in (2.6) follow the normal distribution, we can obtain maximum likelihood estimators of $\beta_{1}$ and $\Sigma$. The definition of scaled partial envelopes does not require the assumption of normality. When normality does not hold, different non-normal error distributions are discussed in the Section 6.

We assume that the data $\left(\left(R_{1 \mid 2}\right)_{i},\left(R_{Y \mid 2}\right)_{i}\right)(i=1, \ldots, n)$ are independent, and $n$ is the sample size. Let $\overline{R_{Y \mid 2}}$ denote the sample mean of $R_{Y \mid 2}$. By means of minimizing the objective function, we can acquire the maximum 
3.2 Maximum likelihood estimators when $\Lambda$ is unknown15

likelihood estimators $\widehat{\Gamma}$ of $\Gamma$ and $\widehat{\Lambda}$ of $\Lambda$. The objective function is as follows

$$
L(\Lambda, \Gamma)=\log \left|\Gamma^{T} \Lambda^{-1} \widetilde{\Sigma}_{\mathrm{res}} \Lambda^{-1} \Gamma\right|+\log \left|\Gamma^{T} \Lambda \widetilde{\Sigma}_{R_{Y \mid 2}}^{-1} \Lambda \Gamma\right| .
$$

More details are provided in the Supplementary Materials. Here, $\widetilde{\Sigma}_{\text {res }}$ denotes the sample covariance matrix of the residuals from the least squares regression of $R_{Y \mid 2}$ on $R_{1 \mid 2}$.

Next, we give the maximum likelihood estimators of the remaining parameters as follows: $\widehat{\Gamma}_{0}$ can be any orthogonal basis of the orthogonal complement of $\operatorname{span}(\widehat{\Gamma}), \widehat{\eta}=\widehat{\Gamma}^{T} \widehat{\Lambda}^{-1} \widetilde{\beta}_{1}, \widehat{\Omega}=\widehat{\Gamma}^{T} \widehat{\Lambda}^{-1} \widetilde{\Sigma}_{\text {res }} \widehat{\Lambda}^{-1} \widehat{\Gamma}, \widehat{\Omega}_{0}=$ $\widehat{\Gamma}_{0}^{T} \widehat{\Lambda}^{-1} \widetilde{\Sigma}_{R_{Y \mid 2}} \widehat{\Lambda}^{-1} \widehat{\Gamma}_{0}, \widehat{\beta}_{1}=\widehat{\Lambda} \widehat{P}_{\Gamma} \widehat{\Lambda}^{-1} \widetilde{\beta}_{1}$, and

$$
\begin{aligned}
\widehat{\Sigma} & =\widehat{\Lambda} \widehat{P}_{\Gamma} \widehat{\Lambda}^{-1} \widetilde{\Sigma}_{\mathrm{res}} \widehat{\Lambda}^{-1} \widehat{P}_{\Gamma} \widehat{\Lambda}+\widehat{\Lambda} \widehat{P}_{\Gamma_{0}} \widehat{\Lambda}^{-1} \widetilde{\Sigma}_{R_{Y \mid 2}} \widehat{\Lambda}^{-1} \widehat{P}_{\Gamma_{0}} \widehat{\Lambda} \\
& =\widehat{\Lambda} \widehat{\Gamma} \widehat{\Omega} \widehat{\Gamma}^{T} \widehat{\Lambda}+\widehat{\Lambda} \widehat{\Gamma}_{0} \widehat{\Omega}_{0} \widehat{\Gamma}_{0}^{T} \widehat{\Lambda}
\end{aligned}
$$

By means of the structures of $\widehat{\beta}_{1}$ and $\widehat{\Sigma}$, we can uncover the course of estimation in the scaled partial envelope model. We think over $\widehat{\beta}_{1}=$ $\widehat{\Lambda} \widehat{P}_{\Gamma} \widehat{\Lambda}^{-1} U^{T} F_{1}\left(F_{1}^{T} F_{1}\right)^{-1}$ where $U$ is the $n \times r$ matrix whose $i$-th row is $\left(\left(R_{Y \mid 2}\right)_{i}-\overline{R_{Y \mid 2}}\right)^{T}$ and $F_{1}$ is the $n \times p_{1}$ matrix whose $i$-th row is $\left(R_{1 \mid 2}\right)_{i}^{T}(i=$ $1, \ldots, n)$. Firstly, the response is rescaled $R_{Y \mid 2} \rightarrow \widehat{\Lambda}^{-1} R_{Y \mid 2}$, and centered to obtain $\widehat{\Lambda}^{-1} U^{T}$. Then, we perform the ordinary partial envelope estimation by employing the rescaled response to obtain $\widehat{P}_{\Gamma} \widehat{\Lambda}^{-1} U^{T} F_{1}\left(F_{1}^{T} F_{1}\right)^{-1}$. After the process above, the estimator is converted back to the original 
scales to obtain $\widehat{\beta}_{1}$. The scaled partial envelope model transforms $R_{Y \mid 2}$ to $\widehat{\Lambda} \widehat{P}_{\Gamma} \widehat{\Lambda}^{-1} R_{Y \mid 2}$, and the process $\widehat{\Lambda} \widehat{P}_{\Gamma} \widehat{\Lambda}^{-1}$ is identical with regarding $\widehat{\Lambda}^{-1}$ as an analogous transformation to the original scale of $\left(R_{Y \mid 2}\right)_{N}$.

\subsection{Parameter identifiability}

There is almost always a unique pair $\{\widehat{\Lambda}, \operatorname{span}(\widehat{\Gamma})\}$ to make the objective function (3.2) to be the global minimizer. When we may discover that $\Lambda$ and $\operatorname{span}(\Gamma)$ are not identified, the objective function will usually be flat along some directions, and can return any value in those directions. The parameters $\beta_{1}$ and $\Sigma$ are our focus of research interest, so it is not a matter for this potential nonuniqueness. In reality, Proposition 1 guarantees that the maximizers of $\beta_{1}$ and $\Sigma$ with reference to the loglikelihood function are solely defined. Then we will obtain the identical estimators $\widehat{\beta}_{1}$ and $\widehat{\Sigma}$, no matter what the global minimizer $\{\widehat{\Lambda}, \operatorname{span}(\widehat{\Gamma})\}$ is unique or not.

According to Henderson and Searle (1979), the operator vec implies that $\mathbb{R}^{a \times b} \rightarrow \mathbb{R}^{a b}$ stacks the columns of a matrix, and the operator vech implies that $\mathbb{R}^{a \times a} \rightarrow \mathbb{R}^{a(a+1) / 2}$ stacks the lower triangular part of a symmetric matrix. Under the circumstances, we associate the constituent parameters $\Lambda, \eta, \Gamma, \Omega$ and $\Omega_{0}$ in the scaled partial envelope models (2.6) with the vector $\phi=\left\{\lambda^{T}, \operatorname{vec}(\eta)^{T}, \operatorname{vec}(\Gamma)^{T}, \operatorname{vech}(\Omega)^{T}, \operatorname{vech}\left(\Omega_{0}\right)^{T}\right\}^{T}=\left(\lambda^{T}, \phi_{o}^{T}\right)^{T}$, where 
$\phi_{o}=\left\{\operatorname{vec}(\eta)^{T}, \operatorname{vec}(\Gamma)^{T}, \operatorname{vech}(\Omega)^{T}, \operatorname{vech}\left(\Omega_{0}\right)^{T}\right\}^{T}$ involves the constituent parameters in the model $(3.1)$ and $\lambda=\left(\lambda_{2}, \ldots, \lambda_{r}\right)^{T}$ is the vector of the 2 nd to the $r$-th diagonal elements of $\Lambda$. Let $A \otimes B$ denote the Kronecker product of matrices $A$ and $B$, and let $L$ denote the $r^{2} \times(r-1)$ matrix with columns $e_{i} \otimes e_{i}$, where $e_{i} \in \mathbb{R}^{r}$ contains a 1 in the $i$-th position and 0 elsewhere, $i=2, \ldots, r$. Then $\lambda=L^{T} \operatorname{vec}(\Lambda)$. As $\beta_{1}=\Lambda \Gamma \eta=\Lambda \beta_{1, o}$ and $\Sigma=\Lambda\left(\Gamma \Omega \Gamma^{T}+\Gamma_{0} \Omega_{0} \Gamma_{0}^{T}\right) \Lambda=\Lambda \Sigma_{o} \Lambda$, both of $\beta_{1}$ and $\Sigma$ are functions of $\phi$

Proposition 1. Suppose that the errors in the scaled partial envelope model (2.6) are independent but not necessarily normal, and have finite second moments, and that $\frac{1}{n} \sum_{i=1}^{n}\left(R_{1 \mid 2}\right)_{i}\left(R_{1 \mid 2}\right)_{i}^{T}>0$. Then $\beta_{1}(\phi)$ and $\Sigma(\phi)$ are identifiable and $\widehat{\beta}_{1}$ and $\widehat{\Sigma}$ are uniquely defined.

Remark 1. From Proposition 1, we can see that when $\phi$ is not identifiable, $\beta_{1}$ and $\Sigma$ are identifiable. Moreover, we can gain unique estimators $\widehat{\beta}_{1}=\beta_{1}(\widehat{\phi})$ and $\widehat{\Sigma}=\Sigma(\widehat{\phi})$. This offers the basis for our discussion of the asymptotic distribution and consistency of $\widehat{\beta}_{1}$ and $\widehat{\Sigma}$ in Section 4 . The proof of Proposition 1 is contained in the Supplementary Materials.

\section{Theoretical properties of the scaled partial envelope estimator}

In this part, we investigate asymptotic distribution and consistency in the scaled partial envelope model. Since the parameters $\beta_{1}$ and 
$\Sigma$ are our primary focus, the asymptotic distribution of the estimator $\left\{\operatorname{vec}\left(\widehat{\beta}_{1}\right)^{T}, \operatorname{vech}(\widehat{\Sigma})^{T}\right\}^{T}$ under normality is provided. To better illustrate the results, we have prepared several definitions below. That is, the contraction matrix $C_{r} \in \mathbb{R}^{r(r+1) / 2 \times r^{2}}$ and the expansion matrix $E_{r} \in \mathbb{R}^{r^{2} \times r(r+1) / 2}$ link the vec and vech operators. For any symmetric matrix $A \in \mathbb{R}^{r \times r}, \operatorname{vec}(A)=$ $E_{r} \operatorname{vech}(A)$ and $\operatorname{vech}(A)=C_{r} \operatorname{vec}(A)$. Let $\Sigma_{R_{1 \mid 2}}=\lim _{n \rightarrow \infty} \frac{1}{n} \sum_{i=1}^{n}\left(R_{1 \mid 2}\right)_{i}\left(R_{1 \mid 2}\right)_{i}^{T}$, and let $p_{1, i i}$ denote the $i$-th diagonal element of the projection matrix $P_{1, F_{1}}$, where $F_{1}$ is the $n \times p_{1}$ matrix whose $i$-th row is $\left(R_{1 \mid 2}\right)_{i}^{T}(i=1, \ldots, n)$. Let $A^{\dagger}$ denote the Moore-Penrose inverse of $A$. The symbol $P_{A(S)}$ denotes the projection in the $S$ inner product onto $A$ or $\operatorname{span}(A)$ if $A$ is a subspace or a matrix, and $Q_{A(S)}=I-P_{A(S)}$. If $\sqrt{n}(\widehat{\theta}-\theta)$ converges to a normal random vector with mean 0 and covariance matrix $V$, we write its asymptotic covariance matrix as $\operatorname{avar}(\sqrt{n} \widehat{\theta})=V$.

The asymptotic covariance matrix in the model (3.1) is written with subscripts ' $O$ '. Next, we represent these formulations. The gradient ma$\operatorname{trix} G_{o}=\partial\left\{\operatorname{vec}\left(\beta_{1, o}\right)^{T}, \operatorname{vech}\left(\Sigma_{o}\right)^{T}\right\}^{T} / \partial \phi_{o}^{T}$ in the model (3.1) has dimension $\left\{p_{1} r+r(r+1) / 2\right\} \times\left\{p_{1} u_{1}+r(r+1) / 2\right\}$ and is expressed as follows $\left(\begin{array}{cccc}I_{p_{1}} \otimes \Gamma & \eta^{T} \otimes I_{r} & 0 & 0 \\ 0 & 2 C_{r}\left(\Gamma \Omega \otimes I_{r}-\Gamma \otimes \Gamma_{0} \Omega_{0} \Gamma_{0}^{T}\right) & C_{r}(\Gamma \otimes \Gamma) E_{u_{1}} & C_{r}\left(\Gamma_{0} \otimes \Gamma_{0}\right) E_{r-u_{1}}\end{array}\right)$

The Fisher information for $\left\{\operatorname{vec}\left(\beta_{1, o}\right)^{T}, \operatorname{vech}\left(\Sigma_{o}\right)^{T}\right\}^{T}$ in the model (3.1) is 
the $\left\{p_{1} r+r(r+1) / 2\right\} \times\left\{p_{1} r+r(r+1) / 2\right\}$ block diagonal matrix

$$
J_{o}=\left(\begin{array}{cc}
\Sigma_{R_{1 \mid 2}} \otimes \Sigma_{o}^{-1} & 0 \\
0 & 2^{-1} E_{r}^{T}\left(\Sigma_{o}^{-1} \otimes \Sigma_{o}^{-1}\right) E_{r}
\end{array}\right) .
$$

Let $h_{o}=\left\{L^{T}\left(\beta_{1, o} \otimes I_{r}\right), 2 L^{T}\left(\Sigma_{o} \otimes I_{r}\right) C_{r}^{T}\right\}^{T}$. We can obtain $h_{o}$ from $h_{o}=\partial\left\{\operatorname{vec}\left(\beta_{1}\right)^{T}, \operatorname{vech}(\Sigma)^{T}\right\}^{T} / \partial \lambda$ in the scaled partial envelope model (2.6) assessed at $\Lambda=I_{r}$. Meanwhile, we have $A_{o}=Q_{G_{o}\left(J_{o}\right)} h_{o} L$ and $D_{\Lambda}=$ $\operatorname{bdiag}\left\{I_{p_{1}} \otimes \Lambda, C_{r}(\Lambda \otimes \Lambda) E_{r}\right\}$ which is a block diagonal matrix and has the identical dimensions with $J_{o}$. Here, all of the quantities which are defined above, merely $D_{\Lambda}$ relies on $\Lambda$.

The gradient matrix $H=\partial\left\{\operatorname{vec}\left(\beta_{1}\right)^{T}, \operatorname{vech}(\Sigma)^{T}\right\}^{T} / \partial \phi^{T}$ in the scaled partial envelope model (2.6) has dimension $\left\{p_{1} r+r(r+1) / 2\right\} \times\left\{r-1+p_{1} u_{1}+\right.$ $r(r+1) / 2\}$, and we can express the gradient matrix as $H=\left\{D_{\Lambda} h_{o}\left(I_{p_{1}} \otimes\right.\right.$ $\left.\left.\Lambda^{-1}\right) L, D_{\Lambda} G_{o}\right\}$. The Fisher information $J$ in the scaled partial envelope model can be acquired by substitute $\Sigma$ for $\Sigma_{o}$ in $J_{o}$, and then we can obtain $J$ as follows

$$
J=\left(\begin{array}{cc}
\Sigma_{R_{1 \mid 2}} \otimes \Sigma^{-1} & 0 \\
0 & 2^{-1} E_{r}^{T}\left(\Sigma^{-1} \otimes \Sigma^{-1}\right) E_{r}
\end{array}\right) .
$$

Proposition 2. Suppose that $\max _{i \leq n} p_{1, i i} \rightarrow 0$ as $n \rightarrow \infty$. Then under model (2.6) which has normal errors, $\sqrt{n}\left[\left\{\operatorname{vec}\left(\widehat{\beta}_{1}\right)-\operatorname{vec}\left(\beta_{1}\right)\right\}^{T},\{\operatorname{vech}(\widehat{\Sigma})-\right.$ $\left.\operatorname{vech}(\Sigma)\}^{T}\right]^{T}$ converges in distribution to a normal random vector with 
mean zero and covariance matrix

$$
\begin{aligned}
V & =H\left(H^{T} J H\right)^{\dagger} H^{T}, \\
& =D_{\Lambda}\left\{A_{o}\left(A_{o}^{T} J_{o} A_{o}\right)^{\dagger} A_{o}^{T}\right\} D_{\Lambda}+D_{\Lambda}\left\{G_{o}\left(G_{o}^{T} J_{o} G_{o}\right)^{\dagger} G_{o}^{T}\right\} D_{\Lambda}, \\
& =V_{1}+V_{2},
\end{aligned}
$$

where $V_{1}=D_{\Lambda}\left\{A_{o}\left(A_{o}^{T} J_{o} A_{o}\right)^{\dagger} A_{o}^{T}\right\} D_{\Lambda}$ and $V_{2}=D_{\Lambda}\left\{G_{o}\left(G_{o}^{T} J_{o} G_{o}\right)^{\dagger} G_{o}^{T}\right\} D_{\Lambda}$.

The proof of Proposition 2 is provided in the Supplementary Materials. Because $J^{-1}-V=J^{-1}-H\left(H^{T} J H\right)^{\dagger} H^{T}=J^{-1 / 2} Q_{J^{1 / 2} H} J^{-1 / 2} \geq 0$, we can obtain $V \leq J^{-1}$, where $J^{-1}$ is the asymptotic covariance matrix of $\left\{\operatorname{vec}\left(\widetilde{\beta}_{1}\right)^{T}, \operatorname{vech}\left(\widetilde{\Sigma}_{\text {res }}\right)^{T}\right\}^{T}$.

We employ the normal likelihood as an objective function to get the scaled partial envelope estimators. When the normality assumption fails, a material question is on the consistency of these estimators. The next proposition provides conditions about $\sqrt{n}$ consistency of $\widehat{\beta}_{1}$ and $\widehat{\Sigma}$.

Proposition 3. Suppose that $\max _{i \leq n} p_{1, i i} \rightarrow 0$ as $n \rightarrow \infty$, the scaled partial envelope model (2.6) has independent but not necessarily normal errors with mean zero and finite fourth moments. Then

$$
\sqrt{n}\left[\left\{\operatorname{vec}\left(\widehat{\beta}_{1}\right)^{T}, \operatorname{vech}(\widehat{\Sigma})^{T}\right\}^{T}-\left\{\operatorname{vec}\left(\beta_{1}\right)^{T}, \operatorname{vech}(\Sigma)^{T}\right\}^{T}\right]
$$

is asymptotically normally distributed, and $\widehat{\beta}_{1}$ and $\widehat{\Sigma}$ are $\sqrt{n}$ consistent estimators of $\beta_{1}$ and $\Sigma$. 
Remark 2. Huber (1973) has built consistency of the standard model estimator $\operatorname{vec}\left(\widetilde{\beta}_{1}\right)$ which requires primarily that the maximum leverage tends to zero as $n \rightarrow \infty$. The assumption about $p_{1, i i}$ has the identical condition with Huber (1973). Furthermore, the estimators are relatively robust to mild deviation from normality in the finite samples. The proof of Proposition 3 is provided in the Supplementary Materials.

\section{Selection of partial envelope dimension $u_{1}$}

In the scaled partial envelope model, we can employ standard techniques such as sequential likelihood-ratio tests (LRT), the Akaike information criterion (AIC) and the Bayesian information criterion (BIC) to select $u_{1}$. Similarly, we also can employ nonparametric methods such as crossvalidation or permutation tests (Cook and Yin, 2001) to choose $u_{1}$.

The BIC estimator of $u_{1}$ is $\arg \min -2 \widehat{L}\left(u_{1}\right)+\log (n) \mathcal{N}\left(u_{1}\right)$, where the minimum ranges from 0 to the integer $r$, and $n$ is the sample size. In the above expression, $\mathcal{N}\left(u_{1}\right)=2 r-1+p_{1} u_{1}+p_{2} r+r(r+1) / 2$ is the number of parameters, and $\widehat{L}\left(u_{1}\right)$ is the maximized loglikelihood in the scaled partial 
envelope model with dimension $u_{1}$,

$$
\begin{aligned}
\widehat{L}\left(u_{1}\right)= & -\frac{n r}{2} \log (2 \pi)-\frac{n}{2} \log \left|\widetilde{\Sigma}_{R_{Y \mid 2}}\right|-\frac{n}{2}\left|\widehat{\Gamma}^{T} \widehat{\Lambda}^{-1} \widetilde{\Sigma}_{\mathrm{res}} \widehat{\Lambda}^{-1} \widehat{\Gamma}\right| \\
& -\frac{n}{2} \log \left|\widehat{\Gamma}^{T} \widehat{\Lambda} \widetilde{\Sigma}_{R_{Y \mid 2}}^{-1} \widehat{\Lambda} \widehat{\Gamma}\right| .
\end{aligned}
$$

Here, $\operatorname{span}(\widehat{\Gamma})$ is the maximum likelihood estimator for $\mathcal{E}_{\Lambda^{-1} \Sigma \Lambda^{-1}}\left(\Lambda^{-1} \mathcal{B}_{1}\right)$ and $\widehat{\Lambda}$ is the maximum likelihood estimator of $\Lambda$ in the scaled partial envelope model. The AIC estimator of $u_{1}$ is $\arg \min -2 \widehat{L}\left(u_{1}\right)+2 \mathcal{N}\left(u_{1}\right)$, and AIC operates analogously.

Properties of AIC and BIC were researched by Cook and $\mathrm{Su}$ (2013, Proposition 4) under the background of response scaling. Similar results are established for the scaled partial envelope model: The candidate set is the set of the scaled partial envelope models having dimensions varying from 0 to $r$. Supposing that there are normal errors in the scaled partial envelope model (2.6), then if there is one and only one true model in the candidate set as $n \rightarrow \infty, \mathrm{BIC}$ will choose the true model with probability approaching 1, and AIC will choose a model that at lowest includes the true model.

\section{Simulation study}

In this section, we carry out the simulation study to compare the scaled partial envelope estimator with the standard model estimator, the 
partial envelope estimator and the scaled envelope estimator in finite sample size. Meanwhile, we employ the algorithm proposed by Cook et al. (2016) for scaled partial envelope estimation, which does not require optimization over a Grassmannian and is displayed to be much faster and typically more accurate than the best existing algorithm proposed by Cook and Zhang (2016). In order to better promote the contrast, we generated data based on the scaled partial envelope model (2.6) following three cases. The first case was $r=10, p=12, p_{1}=8, u=3$ and $u_{1}=2$, the second case was $r=10, p=10, p_{1}=6, u=5$ and $u_{1}=3$, and the third case was $r=10$, $p=6, p_{1}=4, u=6$ and $u_{1}=2$. The elements in $X_{1}$ were generated as independent $N\left(0, p_{1}\right)$ random variables, and the elements in $X_{2}$ were generated as independent $N\left(0, p-p_{1}\right)$ random variables. The matrix $\beta_{2}$ was generated as independent $N\left(r, p-p_{1}\right)$ random variables. We took $\Omega=\sigma^{2} I_{u_{1}}$ and $\Omega_{0}=\sigma_{0}^{2} I_{r-u_{1}}$. The matrix $\eta$ was generated as a $u_{1} \times p_{1}$ matrix of independent $N(0,2)$ random variables, and $\Gamma$ was gained by orthogonalizing an $r \times u_{1}$ matrix of independent $U(0,1)$ random variables. The scale matrix $\Lambda$ was a diagonal matrix with diagonal elements $2^{0}, 2^{0.5}, 2^{1}, 2^{1.5}, \ldots, 2^{4.5}$. We took $\sigma^{2}$ as 0.25 and $\sigma_{0}^{2}$ as 5 . The sample sizes were 100, 300, 600, 1000, 1500 respectively, and we produced 1000 replicates for each sample size. For each sample size, we calculated the standard deviation of each element 
in $\widehat{\beta}_{1}$ over the replicates, and called it the actual standard deviations of the elements in $\widehat{\beta}_{1}$. Also, we calculated the bootstrap standard deviations by means of bootstrapping the residuals 1000 times.

With the above parameter settings, we then fitted four models: (1.1), (2.4), (2.5) and (2.6) to the data separately. Figure 1 plots the standard deviations of a chosen element in $\widehat{\beta}_{1}$ for three different situations where the error is normally distributed when $u<r$. As the sample size increases, all of the standard deviations show a downward trend as a whole, and the efficiency gain increases effectively. In Figure 1, we see an improvement over the ordinary least squares estimators by the partial envelope estimators, and an improvement over the partial envelope estimators by the scaled envelope estimators. When $u<r$, in all three cases, the scaled partial envelope estimators have an obvious improvement over the ordinary least squares estimators and the partial envelope estimators. There are analogous performance between the scaled partial envelope estimators and the scaled envelope estimators in such a situation, where they have the same parameters $\sigma^{2}$ and $\sigma_{0}^{2}$ and the part of $Y$ that is material to predictors is much less variable than the immaterial part whether it is for all predictors or part of predictors. In either case, the bootstrap standard deviation is a good approximative estimator of the actual standard deviation. 
In the above three parameter settings, except for letting $u=r=10$, other parameter settings are unchanged. When $u=r$, the scaled envelope model reduces to the standard multivariate linear regression model abbreviated as the standard model, so there is no probability for efficiency acquisitions in this setting, but we can employ the scaled partial envelope model and still get efficiency gains as long as $p_{1}<r$. In this sense, the scaled partial envelope model is more flexible than the scaled envelope model. Figure 2 plots the standard deviations of a chosen element in $\widehat{\beta}_{1}$ for three different situations with normal error when $u=r$. From Figure 2, we find that the standard model and the scaled envelope model have the same standard deviations, and see that the scaled partial envelope estimators have a remarkable efficiency gain over the partial envelope estimators, and the partial envelope estimators have an obvious efficiency gain over the ordinary least squares estimators and the scaled envelope estimators.

From Table 1, we display the mean and standard deviation of 1000 estimated scales with $\sigma_{0}^{2}=5$ in the $r<p$ situation when $u<r$, and the results in the $r \geq p$ situation are analogous to the $r<p$ situation. Meanwhile, we discover that our algorithm is relatively stable.

Figure 3 exhibits the asymptotic action of the scaled partial envelope estimators under non-normal errors when $u<r$, with the comparison to the 
Table 1: Mean of base 2 logarithms of the diagonal elements in $\widehat{\Lambda}$, the numbers in parentheses are their standard deviations.

\begin{tabular}{|cccc|}
\hline $\mathrm{n}$ & 100 & 600 & 1500 \\
\hline $\log _{2}\left(\widehat{\lambda}_{2}\right)$ & $0.5012(0.0640)$ & $0.5022(0.0241)$ & $0.5015(0.0318)$ \\
$\log _{2}\left(\widehat{\lambda}_{3}\right)$ & $1.0002(0.0856)$ & $1.0030(0.0260)$ & $1.0003(0.0448)$ \\
$\log _{2}\left(\widehat{\lambda}_{4}\right)$ & $1.5012(0.1185)$ & $1.5033(0.0385)$ & $1.5003(0.0664)$ \\
$\log _{2}\left(\widehat{\lambda}_{5}\right)$ & $2.0017(0.2551)$ & $2.0024(0.0766)$ & $2.0005(0.0940)$ \\
$\log _{2}\left(\widehat{\lambda}_{6}\right)$ & $2.5065(0.4660)$ & $2.5035(0.4651)$ & $2.4993(0.1268)$ \\
$\log _{2}\left(\widehat{\lambda}_{7}\right)$ & $3.0028(0.2971)$ & $3.0028(0.2312)$ & $3.0039(0.1854)$ \\
$\log _{2}\left(\widehat{\lambda}_{8}\right)$ & $3.5031(0.4375)$ & $3.5045(0.1948)$ & $3.5000(0.2564)$ \\
$\log _{2}\left(\widehat{\lambda}_{9}\right)$ & $4.0006(0.8645)$ & $4.0026(0.2501)$ & $4.0000(0.3702)$ \\
$\log _{2}\left(\widehat{\lambda}_{10}\right)$ & $4.5033(0.9594)$ & $4.5018(0.3681)$ & $4.5000(0.5038)$ \\
\hline
\end{tabular}

standard model estimators, the partial envelope estimators and the scaled envelope estimators. We implemented the simulations using the identical setup as that in the first case of Figure 1, but we employed a centered t distribution with 6 degrees of freedom, a centered uniform $(0,1)$ distribution and a chi-square distribution with 4 degrees of freedom to represent the distributions with heavier tails, shorter tails, and skewness. Figure 3 does not display obvious differences given rise to the different error distribu- 
tions, so we draw the conclusion that moderate departures from normality do not materially influence the performance of the scaled partial envelope. When the errors obey non-normal distribution, the estimator is no longer a maximum likelihood estimator, but efficiency acquisitions are also still accomplished. Meanwhile, the bootstrap standard deviation is still a good approximative estimator of the actual standard deviation. More importantly, the performance of the scaled partial envelope estimators is significantly better than that of the standard model estimators and the partial envelope estimators. The scaled partial envelope estimators and the scaled envelope estimators also have similar behavior when they have the same parameters $\sigma^{2}$ and $\sigma_{0}^{2}$ and the part of $Y$ that is material to predictors is much less variable than the immaterial part whether it is for all predictors or part of predictors.

Figure 4 displays the asymptotic action of the scaled partial envelope estimators under non-normal errors when $u=r$, with the comparison to the standard model estimators, the partial envelope estimators and the scaled envelope estimators. We implemented the simulations using the identical setup as that in the first case of Figure 2, but we employed a centered t distribution with 6 degrees of freedom, a centered uniform $(0,1)$ distribution and a chi-square distribution with 4 degrees of freedom to represent 
the distributions with heavier tails, shorter tails, and skewness. Through Figure 4, we can find that when $u=r$ and the errors obey non-normal distribution, the standard model and the scaled envelope model have the same standard deviations, and see that the scaled partial envelope estimators have a remarkable efficiency gain over the partial envelope estimators, and the partial envelope estimators have a distinct efficiency gain over the ordinary least squares estimators and the scaled envelope estimators. This agrees with the performance under the normal error.

\section{Real data analysis}

This section is devoted to an example that illustrates advantages of the scaled partial envelope model. The dataset is from Johnson and Wichern (2007) and is on properties of pulp fibers and the paper made from them. The data has 62 measurements on four paper properties: breaking length, elastic modulus, stress at failure and burst strength. The predictors are three properties of fiber: arithmetic fiber length, long fiber fraction and fine fiber fraction. We consider how pulp fiber properties $X$ affects paper properties $Y$, yielding $r=4, p=3$ and $p_{1}=1$. Fine fiber fraction is assigned to the main predictor. Arithmetic fiber length and long fiber fraction are assigned to the covariates. 
We compared the standard errors of the scaled partial envelope estimator $\widehat{\beta}_{1}$ with the standard errors of the ordinary least squares estimator $\widetilde{\beta}_{1}$ by employing the fractions $f_{1, i j}=1-\widehat{\operatorname{avar}}^{1 / 2}\left(\sqrt{n} \widehat{\beta}_{1, i j}\right) / \widehat{\operatorname{avar}}^{1 / 2}\left(\sqrt{n} \widetilde{\beta}_{1, i j}\right)$, where the subscripts $i, j$ show the elements of the estimator of $\beta_{1}$. The standard errors of the ordinary partial envelope estimator and the ordinary least squares estimator were compared in the same manner. Meanwhile, we compared the standard errors of the scaled envelope estimator $\widehat{\beta}$ with the standard errors of the ordinary least squares estimator $\widetilde{\beta}$ by using the fractions $f_{i j}=1-\widehat{\operatorname{avar}}^{1 / 2}\left(\sqrt{n} \widehat{\beta}_{i j}\right) / \widehat{\operatorname{avar}}^{1 / 2}\left(\sqrt{n} \widetilde{\beta}_{i j}\right)$, where the subscripts $i$, $j$ show the elements of the estimator of $\beta$. It is well-known (Shao, 1997; Yang, 2005) that BIC behaves better than AIC if the true model has a simple limited dimensional construction. If someone is concerned more about the estimation bias of the associated envelope model itself over prediction, AIC is more advantageous because it is more conservative when choosing the dimensions. Under this circumstance, we mainly consider $u$ and $u_{1}$ from BIC.

We first fitted the scaled envelope model to all the predictors and BIC suggested that $u=2$. Compared to $\widetilde{\beta}$, the standard deviations of the elements in the scaled envelope estimator were $0.15 \%$ to $1.97 \%$ smaller, $0.0015 \leq f_{i j} \leq 0.0197$. A sample size of about $n=65$ observations would be 
needed to reduce the standard error of the ordinary least squares estimator by 1.97 , so employing the scaled envelope estimator is roughly equal to the sample size for inference on some elements of $\beta$ with the ordinary least squares estimator. This implies that the scaled envelope model does not obtain much efficiency comparing to the standard model. The reason roots in the estimated structure of $\Sigma$ : the eigenvalues of $\widehat{\Sigma}_{\mathcal{E}}$ are 6.6441 and 0.0176 while the eigenvalues for $\widehat{\Sigma}_{\mathcal{E}^{\perp}}$ are 0.1310 and 0.0109 . So the part of $Y$ that is material to $X$ is no less variable than the immaterial part, and we do not acquire much efficiency from the scaled envelope model.

Next we fitted an ordinary partial envelope model to the data and BIC suggested that $u_{1}=1$. Compared to $\widetilde{\beta}_{1}$, the standard deviations of the elements in the ordinary partial envelope estimator were $61.62 \%$ to $87.46 \%$ smaller, $0.6162 \leq f_{1, i j} \leq 0.8746$. Therefore, employing the ordinary partial envelope estimator is roughly equal to being 64 times the sample size for inference on some elements of $\beta_{1}$ with the ordinary least squares estimator.

When the scaled partial envelope model was fitted to the data, BIC suggested that $u_{1}=1$. The scale transformation matrix $\Lambda$ was estimated with diagonal elements $1,2^{0.5}, 2^{1}, 2^{1.5}$. Compared to $\widetilde{\beta}_{1}$, the standard deviations of the elements in the scaled partial envelope estimator were $44.62 \%$ to $99.10 \%$ smaller, $0.4462 \leq f_{1, i j} \leq 0.9910$, which is a significant improve- 
ment over the gains provided by the scaled partial envelope model. In other words, a sample size of about $n=12 \times 10^{3}$ observations would be needed to reduce the standard error of the ordinary least squares estimator by 99.10 . Because the part of $Y$ that is material to this predictor is much less variable than the immaterial part, there is substantial reduction achieved when we pay close attention to fine fiber fraction. Focusing on the estimated struc-

ture of $\widehat{\Sigma}, \widehat{\Sigma}_{\mathcal{E}_{1}}$ has eigenvalue 0.00007 while $\widehat{\Sigma}_{\mathcal{E}_{1}^{\perp}}$ has eigenvalues 3.9521 , 0.0145 and 0.00002 . In general, when the part of $Y$ that is material to all predictors is no less variable than the immaterial part, but the part of $Y$ that is material to part of predictors is much less variable than the immaterial part, the scaled partial envelope estimators have significant advantage over the scaled envelope estimators.

\section{Concluding remarks}

In this paper, we extend the partial envelope model to the scaled partial envelope model to reduce dimension efficiently and keep scale invariable. Then, we give the maximum likelihood estimation and parameter identifiability. Meanwhile, we demonstrate theoretical properties and selection of partial envelope dimension. Simulation studies are carried out to compare the proposed scaled partial envelope model with the standard model, the 
partial envelope model and the scaled envelope model. At the same time, real data example is also analysed for exhibiting superiorities of the scaled partial envelope model. By means of introducing a scaling parameter for each response variable, the scaled partial envelope estimator widens the effective range of partial envelope structures, and can bring efficiency gains that are not provided by the ordinary partial envelope estimator.

We consider the case where the predictor and response variables in the scaled partial envelope model are vector-valued in this article. Interesting topics for further studies contain investigating the case where the predictor and response variables in the model are extended to functional data or matrix-valued. Meanwhile, the techniques in this paper can be applied to other settings, such as tensor regression and discriminant analysis. If prior information emerges, a Bayesian version of this model is satisfying. The identical idea and techniques can be extended to semiparametric settings, such as quantile regression and expectile regression.

\section{Acknowledgements}

This research was supported by the National Natural Science Foundation of China (Grant Nos. 11471160, 11101114), the National Statistical Science Research Major Program of China (Grant No. 2018LD01), the Fundamental Research Funds for the Central Universities, PR China (Grant 
Nos. 30920130111015), sponsored by Qing Lan Project and a grant from the University Grants Council of Hong Kong, China. The authors thank for the editor, the associate editor and two referees for their suggestions that led to the improvement of an early manuscript.

\section{References}

Adragni, K. P., Cook, R. D. and Wu, S. (2012). Grassmannoptim: An R package for Grassmann manifold optimization. Journal of Statistical Software 50, 1-18.

Cook, R. D., Forzani, L. and Su, Z. (2016). A note on fast envelope estimation. Journal of Multivariate Analysis 150, 42-54.

Cook, R. D., Helland, I. S. and Su, Z. (2013). Envelopes and partial least squares regression. Journal of the Royal Statistical Society: Series B (Statistical Methodology) 75, 851-877.

Cook, R. D., Li, B. and Chiaromonte, F. (2010). Envelope models for parsimonious and efficient multivariate linear regression. Statistica Sinica 20, 927-1010.

Cook, R. D. and Su, Z. (2013). Scaled envelopes: scale-invariant and efficient estimation in multivariate linear regression. Biometrika 100, 939-954.

Cook, R. D. and Su, Z. (2016). Scaled predictor envelopes and partial least-squares regression. Technometrics 58, 155-165.

Cook, R. D. and Yin, X. (2001). Dimension reduction and visualization in discriminant analysis (with discussion). Australian and New Zealand Journal of Statistics 43, 147-199. 


\section{REFERENCES34}

Cook, R. D. and Zhang, X. (2015). Foundations for envelope models and methods. Journal of the American Statistical Association 110, 599-611.

Cook, R. D. and Zhang, X. (2016). Algorithms for envelope estimation. Journal of Computational and Graphical Statistics 25, 284-300.

Cook, R. D. and Zhang, X. (2018). Fast envelope algorithms. Statistica Sinica 28, 1179-1197.

Ding, S. and Cook, R. D. (2018). Matrix variate regressions and envelope models. Journal of the Royal Statistical Society: Series B (Statistical Methodology) 80, 387-408.

Edelman, A., Arias, T. A. and Smith, S. T. (1998). The geometry of algorithms with orthogonality constraints. SIAM journal on Matrix Analysis and Applications 20, 303-353.

Henderson, H. V. and Searle, S. R. (1979). Vec and vech operators for matrices, with some uses in Jacobians and multivariate statistics. Canadian Journal of Statistics 7, 65-81.

Huber, P. J. (1973). Robust regression: asymptotics, conjectures and Monte Carlo. The Annals of Statistics 1, 799-821.

Johnson, R. A. and Wichern, D. W. (2007). Applied Multivariate Statistical Analysis(6 ed.). Upper Saddle River, NJ: Prentice Hall.

Khare, K., Pal, S. and Su, Z. (2017). A bayesian approach for envelope models. The Annals of Statistics 45, 196-222.

Li, L. and Zhang, X. (2017). Parsimonious tensor response regression. Journal of the American Statistical Association 112, 1131-1146. 


\section{REFERENCES35}

Pan, Y., Mai, Q. and Zhang, X. (2019). Covariate-adjusted tensor classification in high dimensions. Journal of the American Statistical Association 114, 1305-1319.

Shao, J. (1997). An asymptotic theory for linear model selection. Statistica Sinica 7, 221-242.

Shapiro, A. (1986). Asymptotic theory of overparameterized structural models. Journal of the American Statistical Association 81, 142-149.

Su, Z. and Cook, R. D. (2011). Partial envelopes for efficient estimation in multivariate linear regression. Biometrika 98, 133-146.

Su, Z. and Cook, R. D. (2012). Inner envelopes: efficient estimation in multivariate linear regression. Biometrika $\mathbf{9 9}, 687-702$.

Su, Z. and Cook, R. D. (2013). Estimation of multivariate means with heteroscedastic errors using envelope models. Statistica Sinica 23, 213-230.

Yang, Y. (2005). Can the strengths of AIC and BIC be shared? A conflict between model indentification and regression estimation. Biometrika 92, 937-950.

Zhang, X. and Li, L. (2017). Tensor envelope partial least-squares regression. Technometrics 59, $426-436$.

Zhu, G. and Su, Z. (2020). Envelope-based sparse partial least squares. The Annals of Statistics 48, 161-182.

${ }^{a}$ School of Science, Nanjing University of Science and Technology, Nanjing, 210094, Jiangsu, P. R. China 


\section{REFERENCES36}

${ }^{b}$ School of Finance, Chuzhou University, Chuzhou, 239000, Anhui, P. R. China

E-mail: s20082112095@163.com

${ }^{a}$ School of Science, Nanjing University of Science and Technology, Nanjing, 210094, Jiangsu, P.

R. China

E-mail: stahzs@126.com

${ }^{c}$ Center for Statistics and Data Science, Beijing Normal University

${ }^{d}$ Department of Mathematics, Hong Kong Baptist University, Kowloon Tong, Hong Kong, China

E-mail: lzhu@hkbu.edu.hk 


\section{REFERENCES37}
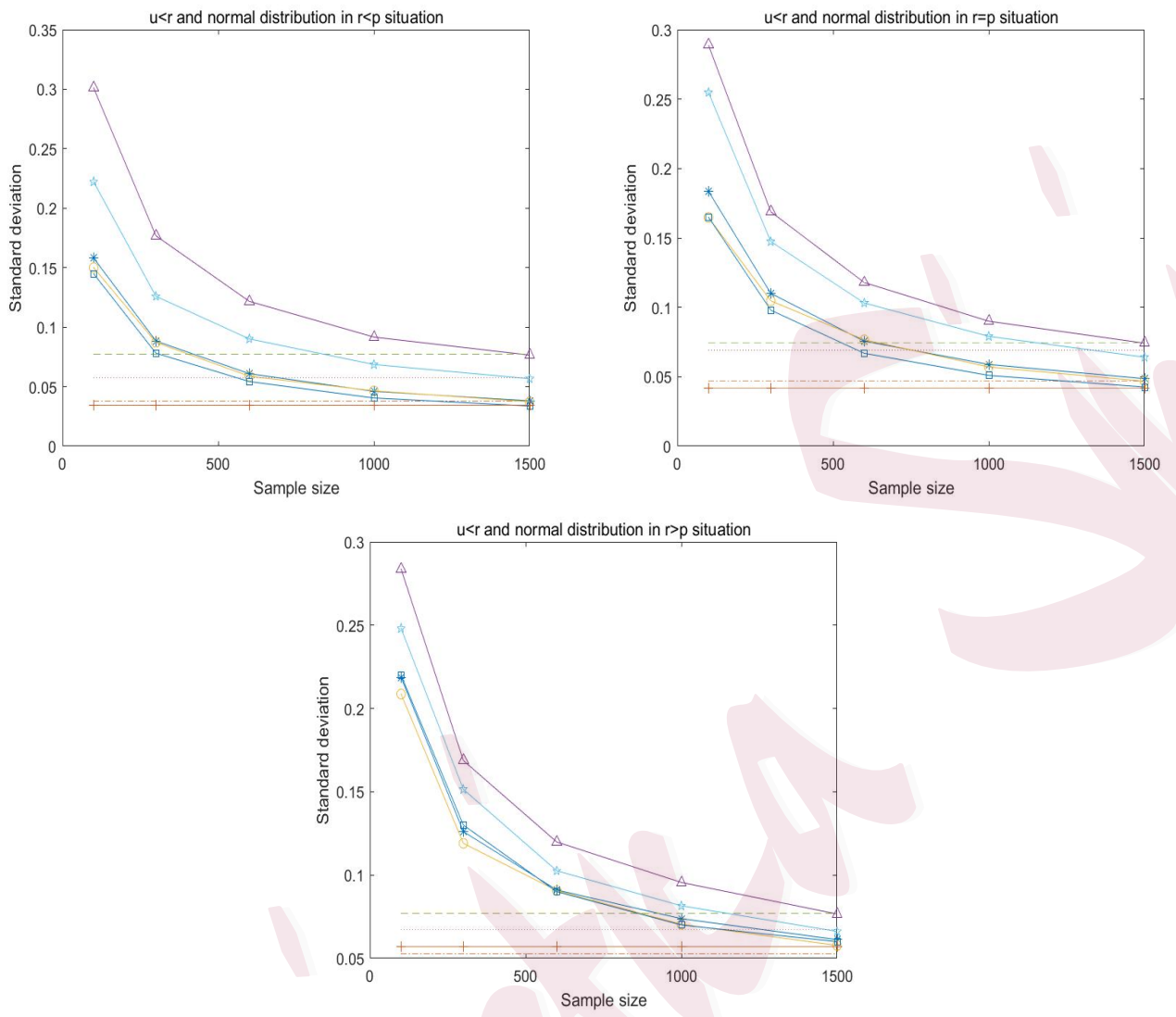

Figure 1: Comparison of the scaled partial envelope estimators, the standard mod-

el estimators, the partial envelope estimators and the scaled envelope estimators: the actual standard deviation of scaled partial envelope estimators by ' $-*$ ', the asymptotic standard deviation of scaled partial envelope estimators by '-.', the bootstrap standard deviation of scaled partial envelope estimators by ' $-\bigcirc$ '; the actual standard deviation of standard model estimators by ' $-\triangle^{\prime}$, the asymptotic standard deviation of standard model estimators by '--'; the actual standard deviation of partial envelope estimators by '- -5 ', the asymptotic standard deviation of partial envelope estimators by '...'; the actual standard deviation of scaled envelope estimators by ' $-\square$ ', the asymptotic standard deviation of scaled envelope estimators by ' -+ '. 


\section{REFERENCES38}
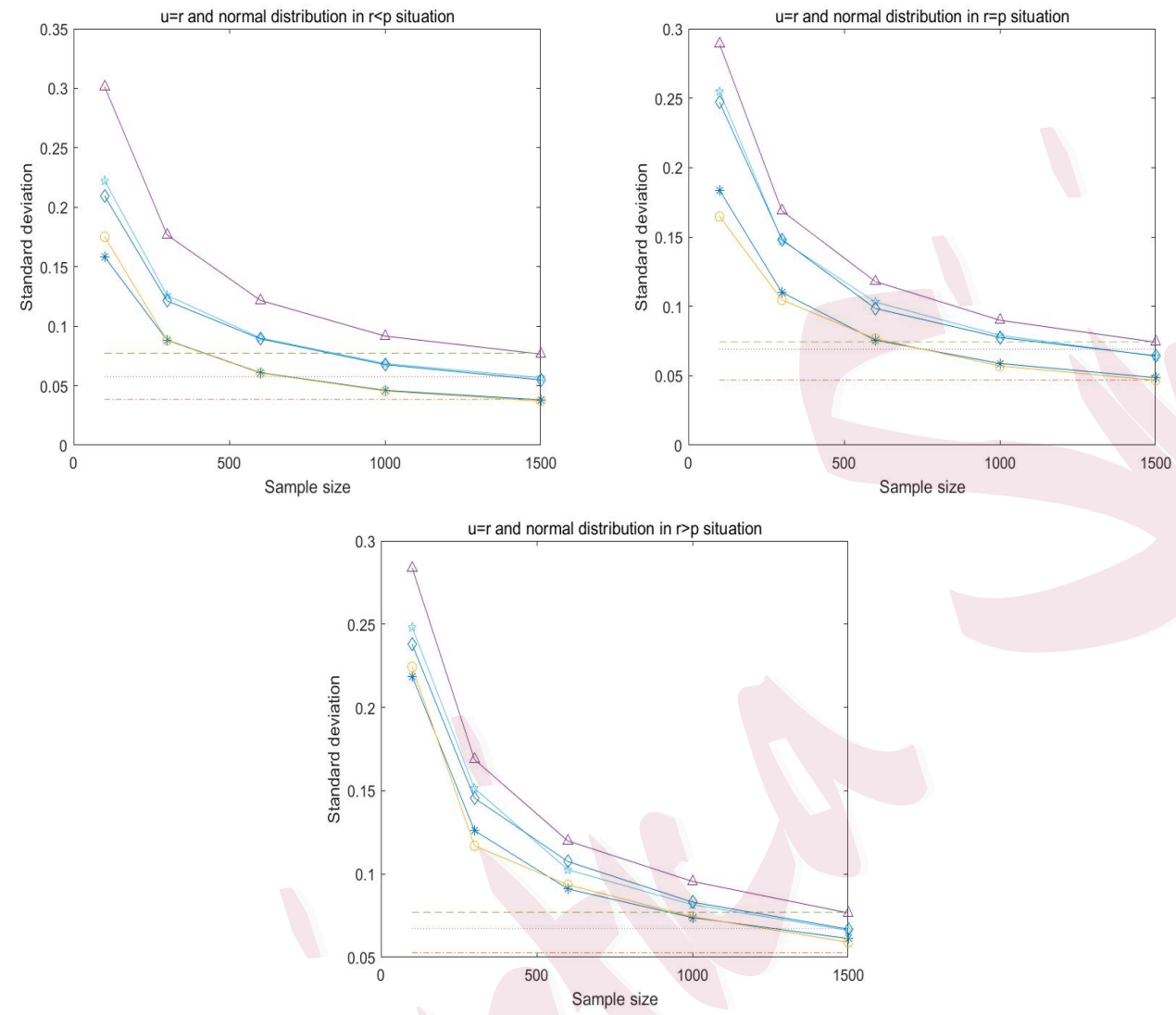

Figure 2: Comparison of the scaled partial envelope estimators, the standard model estimators and the partial envelope estimators: the actual standard deviation of scaled partial envelope estimators by ' $-*$ ', the asymptotic standard deviation of scaled partial envelope estimators by '.- ', the bootstrap standard deviation of scaled partial envelope estimators by ' $-\bigcirc$ '; the actual standard deviation of standard model estimators by ' $-\triangle$ ', the asymptotic standard deviation of standard model estimators by '--'; the actual standard deviation of partial envelope estimators by ' -5 ', the asymptotic standard deviation of partial envelope estimators by '...', the bootstrap standard deviation of partial envelope estimators by ' $-\checkmark$ '. 

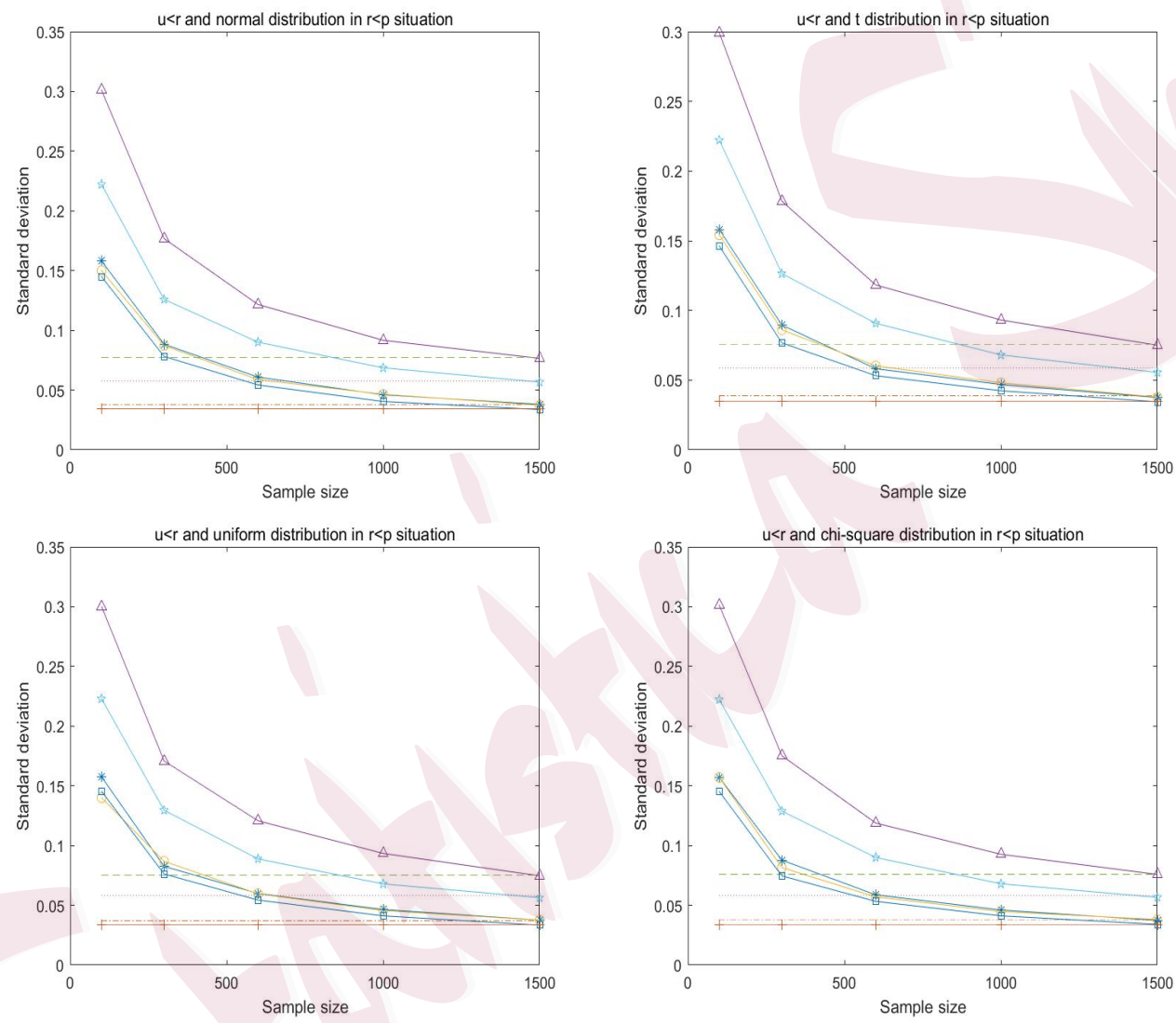

Figure 3: Comparison of the scaled partial envelope estimators with normal, $t_{6}$, $U(0,1)$ and $\chi_{4}^{2}$ errors when $u<r$. The line marks are the same as those in Figure 1. 

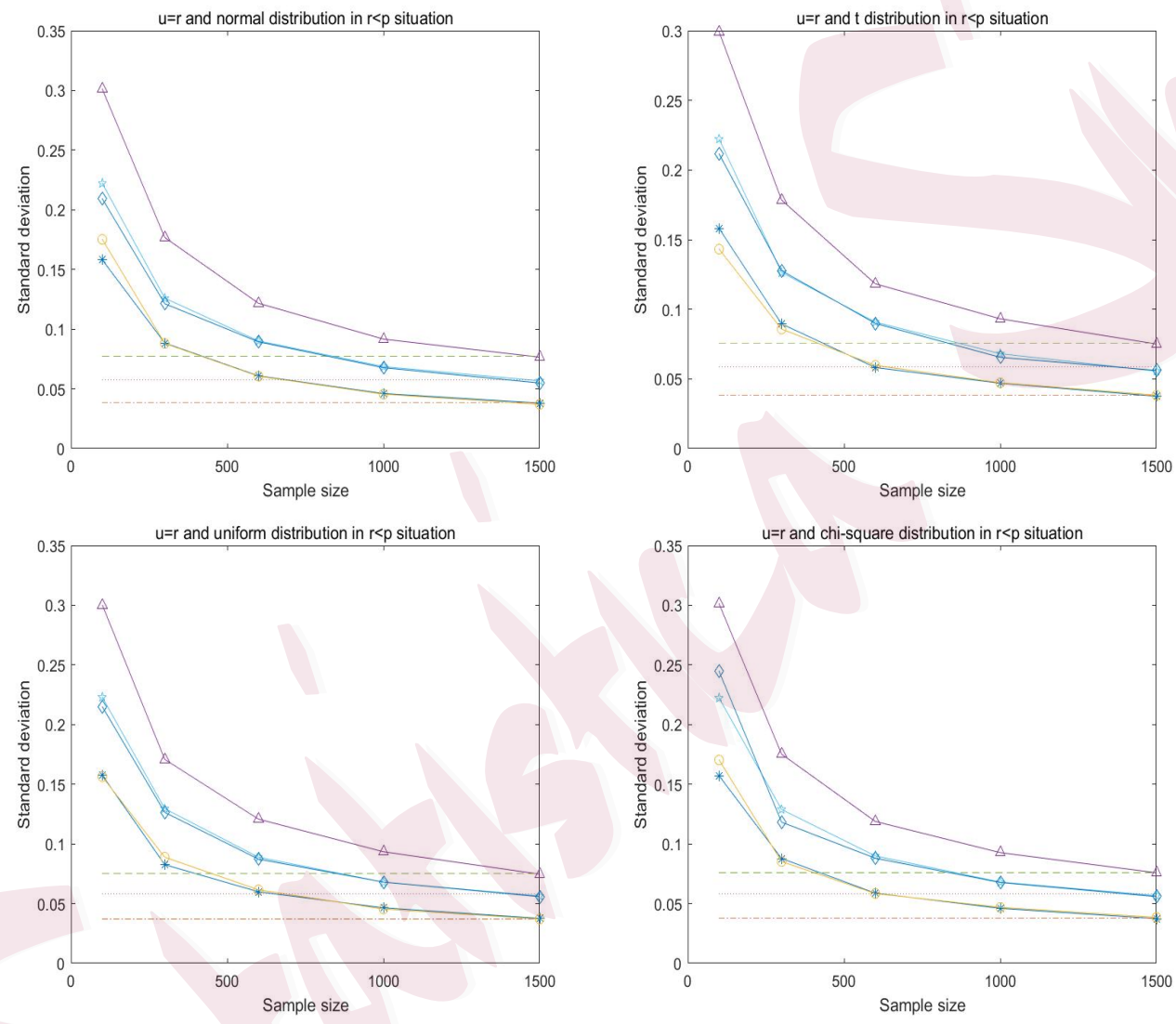

Figure 4: Comparison of the scaled partial envelope estimators with normal, $t_{6}$, $U(0,1)$ and $\chi_{4}^{2}$ errors when $u=r$. The line marks are the same as those in Figure 2. 